\title{
A SURVEY OF QOS ROUTING PROTOCOLS FOR MOBILE AD HOC NETWORKS
}

\author{
G. Santhi ${ }^{1}$ and Alamelu Nachiappan ${ }^{2}$ \\ ${ }^{1}$ Department of IT, Pondicherry Engineering College, India. \\ shanthikarthikeyan@pec.edu \\ ${ }^{2}$ Department of EEE, Pondicherry Engineering College, India. \\ nalam63eyahoo.com
}

\begin{abstract}
A Mobile Ad-hoc Network (MANET) is composed of mobile nodes without any infrastructure. MANET applications such as audio/video conferencing, webcasting requires very stringent and inflexible Quality of Service (QoS). The provision of QoS guarantees is much more challenging in MANETs than wired networks due to node mobility, limited power supply and a lack of centralized control. Many researches have been done so as to provide QoS assurances by designing various MANET protocols. In recent years a number of QoS routing protocols with distinguishing features have been newly proposed. However, systematic performance evaluations and comparative analysis of these protocols in a common realistic environment have been performed only in a limited manner. This paper presents a thorough overview of QoS routing metrics, resources and factors affecting performance of QoS routing protocols. The relative strength, weakness, and applicability of existing QoS routing protocols are also studied and compared. QoS routing protocols are classified according to the QoS metrics used, type of QoS guarantee assured and their interaction with the medium access control (MAC) protocol.
\end{abstract}

KEYWORDS: MANETs, Quality of Service, Routing protocol, mobile node, Taxonomy.

\section{INTRODUCTION}

A MANET is a self-organizing collection of wireless mobile nodes that form a temporary and dynamic wireless network without any infrastructure. MANETs are self-configuring; there is no central management system with configuration responsibilities. All the mobile nodes can communicate each other directly, if they are in other's wireless links radio range. In order to enable data transfer they either communicate through single hop or through multiple hops with the help of intermediate nodes. Since MANETs allow ubiquitous service access, anywhere, anytime without any fixed infrastructure they can be widely used in military battlefields, crisis management services, classrooms and conference halls etc. MANETs ad-hoc fashion networking developments lead to development of enormous multimedia applications such as video-on-demand, video conferencing etc.

Quality of Service (QoS) is the performance level of a service offered by the network to the user. Most of the multimedia applications have stringent QoS requirements that must be satisfied. The goal of QoS provisioning is to achieve a more deterministic network behavior, so that information carried by the network can be better delivered and network resources can be better utilized. However, there still remains a significant challenge to provide QoS solutions and maintain end-to-end QoS with user mobility.

Most of the conventional routing protocols are designed either to minimize the data traffic in the network or to minimize the average hops for delivering a packet. [1]. Even some protocols 
such as Ad-hoc On demand Distance Vector (AODV) [2], Dynamic Source Routing (DSR) [3] and On-demand Multicast Routing Protocol (ODMRP) [4] are designed without explicitly considering QoS. When QoS is considered, some protocols may be unsatisfactory or impractical due to the lack of resources and the excessive computation overhead. QoS routing usually involves two tasks: collecting and maintaining up-to-date state information about the network and finding feasible paths for a connection based on its QoS requirements. [5] To support QoS, a service can be characterized by a set of measurable pre specified service requirements such as minimum bandwidth, maximum delay, maximum delay variance and maximum packet loss rate. However many other metrics are also used to quantify QoS and in this paper we cover most of them in detail.

The remainder of this paper is organized as follows. In Section II, we discuss related works in terms of QoS routing surveys and summarize their main points. Review of the several challenges faced by the provision of QoS on the MANET environment is given is section III. In section IV, we analyze the QoS routing metrics commonly used by all applications and the tradeoffs involved in the protocol design. Section V presents the taxonomy of QoS routing protocols based on their network architecture, type of QoS guarantee assured and the interaction with the MAC layer. Following this, we summarize and compare the operations, key features and major advantages and drawbacks of a selection of QoS routing protocols proposed in the literature. Finally we draw the conclusions and discuss our findings in the field of QoS routing.

\section{RELATED WORKS}

Routing protocols belonging to different QoS philosophies have been proposed in the literature. A fairly comprehensive overview of the state of the field of QoS in networking was provided by Chen in 1999 [6]. Chakrabarti and Mishra [7] later summarized the important QoS related issues in MANETs in 2001 and their conclusions highlighted several significant points in MANET research. It includes admission control policies and protocols, QoS robustness and QoS preservation under failure conditions.

In 2004, Al-Karaki and Kamal published a detailed overview [8] and the development trends in the field of QoS routing. They highlighted some areas such as security and multicast routing requiring further research attention. They were categorized the QoS routing solutions into various types of approaches: Flat, Hierarchical, Position-based and power aware QoS routing.

Reddy et al. [9] provided a thorough overview of the more widely accepted MAC and routing solutions for providing better QoS in MANETs.

\section{ISSUES AND CHALLENGES WHILE PROVIDING QOS IN AD-HOC NETWORKS}

QoS provision will lead to an increase in computational and communicational cost. In other words, it requires more time to setup a connection and maintains more state information per connection. The improvement in network utilization counterbalances the increase in state information and the associated complexity and various issues are needed to be faced while providing QoS for MANETS. The major problems that are faced are as follows:

Unreliable channel: The bit errors are the main problem which arises because of the unreliable wireless channels. These channels cause high bit error rate and this is due to high interference, thermal noise, multipath fading effects, [10] and so on. This leads to low packet delivery ratio. 
Since the medium is wireless in the case of MANETs, it may also lead to leakage of information into the surroundings.

Maintenance of route: The dynamic nature of the network topology and changing behavior of the communication medium makes the maintenance of network state information very difficult. The established routing paths may be broken even during the process of data transfer. Hence the need for maintenance and reconstruction of routing paths with minimal overhead and delay causes. The QoS aware routing would require the reservation of resources at the intermediate nodes. The reservation maintenance with the changes in topology becomes cumbersome.

Mobility of the node: Since the nodes considered here are mobile nodes, that is they move independently and randomly at any direction and speed, the topology information has to be updated frequently and accordingly so as to provide routing to reach the final destination which result in again less packet delivery ratio. [11]

Limited power supply: The mobile nodes are generally constrained by limited power supply compared to nodes in the wired networks. Providing QoS consumes more power due to overhead from the mobile nodes which may drain the node's power quickly.

Lack of centralized control: The members of any ad hoc networks can join or leave the network dynamically and the network is set up spontaneously. So there may not be any provision of centralized control on the nodes which leads to increased algorithm's overhead and complexity, as QoS state information must be disseminated efficiently.

Channel contention: Nodes in a MANET must communicate with each other on a common channel so as to provide the network topology. However, this introduces the problems of interference and channel contention. For peer-to-peer data communications these can be avoided in various ways. One way is to attempt global clock synchronization and use a TDMA-based system where each node may transmit at a predefined time. This is difficult to achieve since there is no centralized control on the nodes. Other ways are to use a different frequency band or spreading code (as in CDMA) for each transmitter. This requires a distributed channel selection mechanism as well as the dissemination of channel information. [12]

Security: Security can be considered as a QoS attribute. Without adequate security, unauthorized accesses and usages may violate the QoS negotiations. The nature of broadcasts in wireless networks potentially results in more security exposures. The physical medium of communication is inherently insecure. So we need to design security-aware routing algorithms for ad hoc networks.

\section{Evaluation Metrics for QoS Routing Protocols}

As different applications have different requirements, the services required by them and the associated QoS parameters differ from application to application. For example, in case of multimedia applications, bandwidth, delay and delay-jitter are the key QoS parameters, whereas military applications have stringent security requirements. The following is a sample of the metrics commonly used by applications to specify QoS requirement to the routing protocol.

- Minimum Throughput (bps) - the desired application data throughput. [13]

- Maximum Delay (s) - maximum tolerable end-to-end delay for data packets. [14] 
- Maximum Delay jitter - difference between the upper bound on end-to-end delay and the absolute minimum delay. [15]

- Maximum Packet loss ratio - the acceptable percentage of total packets sent, which arnot received by the final destination node. [16]

QoS metrics such as above can be classified as additive, concave or multiplicative metrics based on their mathematical properties. [9]. An additive metrics are defined as $\sum_{i=1}^{n} \operatorname{Li}(m)$ over path $\mathrm{P}$ of length $\mathrm{n}$, where $\mathrm{L}_{\mathrm{i}}(\mathrm{m})$ is the value of metric $\mathrm{m}$ over link $\mathrm{L}_{\mathrm{i}}$ and $\mathrm{L}_{\mathrm{i}} \in \mathrm{P}$. A concave metric represents the minimum value over a path $\mathrm{P}$ and is formally defined as $\mathrm{C}_{\mathrm{m}}=\min (\operatorname{Li}(\mathrm{m}))$. Finally a multiplicative metric $\mathbf{M}_{\mathrm{m}}$ is calculated by taking the product of the values along a path i.e. $\mathrm{M}_{\mathrm{m}}=\prod_{i=1}^{n} \operatorname{Li}(m)$. Thus, end-to-end delay for example, is an additive metric, since it is cumulative over the whole path. Bandwidth is a concave metric, since we are only interested in the bottleneck: the minimum value on the path. Finally, path reliability is a multiplicative metric, since the reliabilities of each link in the path must be multiplied together to compute the chance of delivering the packet via a given route. An application may typically request a particular quality of service by specifying its requirements in terms of one or more of the above metrics.

\section{Classification of QoS Routing Protocols}

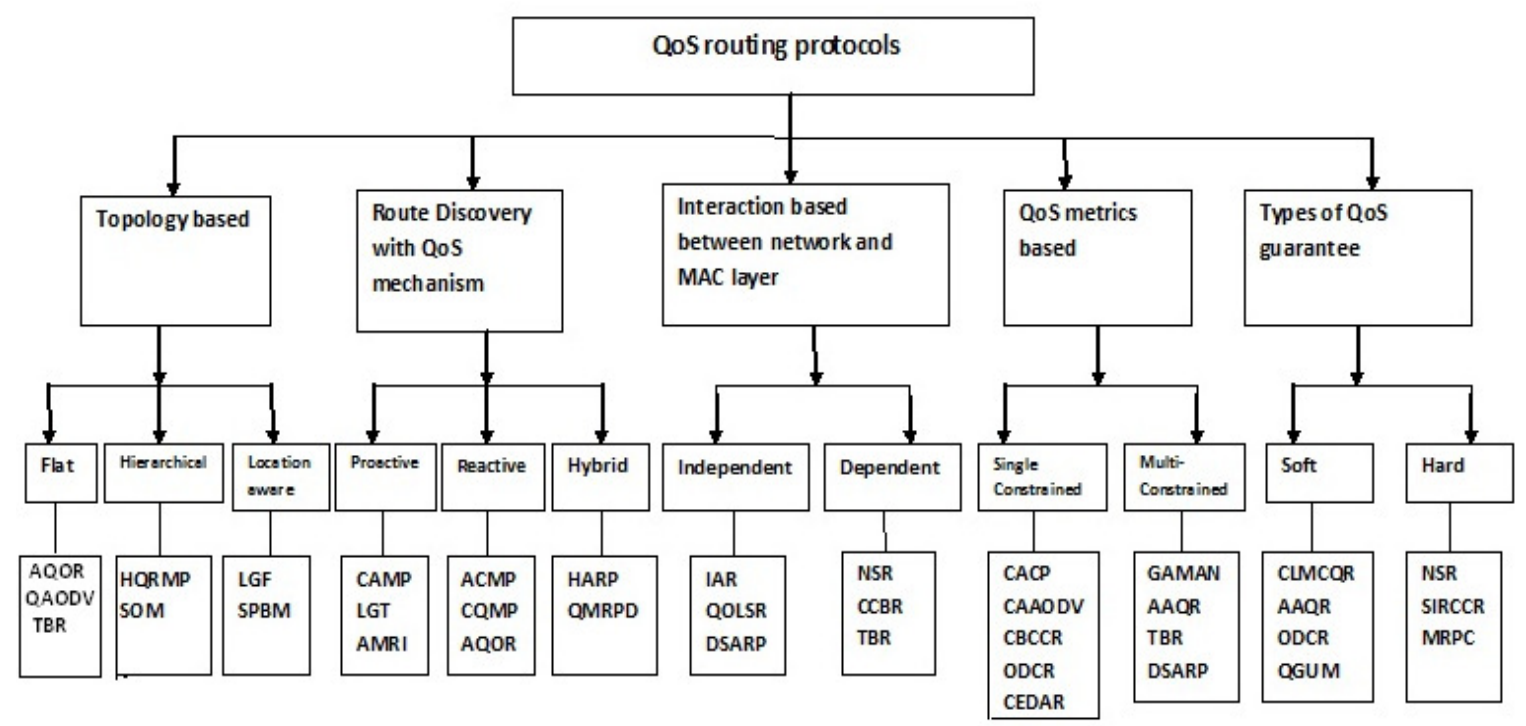

Figure 1. Taxonomy of QoS routing protocols

J.N. Al-Karaki et.al., [8] classified the QoS routing protocols based on their

- Network topology (Flat, Hierarchical and Location-aware),

- An approach to route discovery with QoS (Proactive, Reactive and Hybrid).

On the other hand Reddy et.al., [9] classified the QoS routing protocols based on the

- interaction between the network and the MAC layer: (Dependent or Independent) 
In this paper, we have considered the following issues for QoS routing solutions.

- QoS metrics focused: (Single constrained or Multi constrained)

- Type of QoS guarantee assured: (Hard or Soft)

\subsection{Network Topology Based Protocols}

One of the most popular methods to distinguish MANETs QoS protocols is based on how distribution paths among group members are constructed. In terms of this method, existing QoS protocols can be divided into flat, hierarchical and hybrid protocols. Most of the routing protocols assume physically flat network architecture with mobile nodes having homogeneous capability in terms of network resources and computing power. In practice however, this assumption may not often hold since there exist various types of mobile nodes with different roles, capacities and mobility patterns.

In an architecture-based multicast routing protocol, MANETs have physically hierarchical architectures, which are formed by different types of mobile nodes. For example, Hierarchical QoS

Multicast Routing Protocol (HQMRP) [17] for MANETs builds a multicast structure at each level of the hierarchy for efficient and scalable multicast message delivery. Self-Organizing Map (SOM) [18] and Core-Extraction Distributed Ad hoc Routing algorithm (CEDAR) [19] is also a typical hierarchical architecture, which provides a way for automatically organizing the hierarchical architecture. In location-based multicast routing protocols, the availability of a Global Positioning System (GPS), Bluetooth or other locations systems easily gets geographical information of mobile nodes when needed. Location-based Geo casting and Forwarding (LGF) [20], LGT [21] and Scalable Position-Based Multicast (SPBM) [22] protocol are typical locationbased QoS routing protocols for MANETs.

\subsection{Route Discovery with QoS based protocols}

Based on the routing information update mechanism employed, QoS approaches can be classified into three categories viz., Proactive, on-demand, and hybrid QoS approaches.

Proactive protocols are one where a routing table is maintained at every node which aids in forwarding packets. These tables are updated regularly in order to maintain up-to-date routing information from each node to every other node. Therefore, the source node can get a routing path immediately if it needs one. There are some typical proactive QoS routing protocols such as QOLSR [23] (QoS Optimized Link State Routing) and PLBQR [24] (Predictive Location-Based QoS Routing in Mobile Ad Hoc Networks).

A reactive protocol is also called "on-demand" protocols. Reactive protocols are one which does not require the maintenance of network topology when there is no traffic. The state information is acquired when needed. However, route maintenance is an important operation of reactive routing protocols, because source nodes may suffer from long delays for route searching before they can forward data packets. QoS AODV [25] (QoS Ad-hoc On demand Distance Vector), ACMP [26] (Adaptive Core based Routing Protocol with Consolidated Query Packets) and CQMP (Mesh-based Multicast Routing Protocol with Consolidated Query Packets) [27] are typical examples for reactive routing protocols. Compared to proactive routing protocols, less control overhead is the significant advantage of the reactive routing protocols. 
A hybrid protocol as the name implies it is a combination of both proactive and reactive strategies. Hence, hybrid protocols address both efficiency and robustness. The Efficient hybrid Multicast Routing Protocol (EHMRP) [28] is an instance for hybrid-based QoS routing protocol.

\subsection{Protocols based on interaction between network and MAC layer}

Based on the interaction with MAC layer, QoS protocols can be classified into two categories, independent and dependent QoS protocols. In the independent QoS protocol, the network layer is not dependent on the MAC layer for QoS provisioning. They typically estimate node or link states and attempt to route using those nodes and links for which more favorable conditions exist. However, the achievable level of performance is usually not quantified or is only relative and therefore no promises can be made to applications. The aim of such protocols is typically to foster a better average QoS for all packets according to one or more metrics. QOLSR (QoS Optimized Link State Routing), DSARP (Delay-Sensitive Adaptive Routing Protocol) [29] and IAR (Interference-Aware Routing) [30] are typical independent protocols.

The dependent QoS protocol requires the MAC layer to assist the routing protocol for QoS provisioning. It performs implicit resource reservation and provides QoS guarantees. Entropybased routing (EBR) [31], Channel Capacity-Based Routing (CCBR) [13] and Node State Routing (NSR) [32] are typical dependent protocols.

\subsection{Single constrained vs Multi constrained QoS metrics}

Most of the protocols focused on providing an assured throughput service only, since throughput was deemed the most important requirement in earlier days. These single-constrained routing protocols have received success in many aspects; however, they do not always perform best. In CEDAR the bandwidth is used as the only QoS parameter for routing.

Most of the multimedia applications require the communication to meet stringent requirements on delay, delay-jitter, cost and other QoS metrics. In this context, the trend is to move from single constrained routing to multi constrained routing. The main function of multiconstrained QoS routing is to find a feasible path that satisfies multiple constraints simultaneously, which is a big challenge for MANETs where the topology may change constantly. It has been proved that such a problem is NP-complete. QMRPD (QoS Multicast Routing Protocol for Dynamic group topology) [33] GAMAN (Genetic Algorithm-based routing for MANETs) [34] HMCOP (Heuristic multi Constrained Optimal Path) are typical multi constrained routing protocols.

\subsection{Hard QoS vs Soft QoS approach}

The QoS provisioning approaches can be broadly classified into two categories, hard QoS and soft QoS approaches. If QoS requirements of a connection are guaranteed to be met for the whole duration of the session, the QoS approach is termed as hard QoS approach. In MANETS it is very challenging to provide hard QoS guarantees to user applications. Some of the protocols NSR and SIRCCR [35] (SIR and Channel Capacity based Routing).

If the QoS requirements are not guaranteed for the entire session, the QoS approach is termed as soft QoS approach. Thus, QoS guarantees can only be given within certain statistical bounds. Most of the protocols provide soft QoS guarantees. 


\section{QoS-aware Routing Protocols}

The primary goal of the QoS-aware routing protocols is to determine a path from a source to the destination that satisfies the needs of the desired QoS. The QoS-aware path is determined within the constraints of bandwidth, minimal search, distance, and traffic conditions. Since path selection is based on the desired QoS, the routing protocol can be termed QoS-aware. In the literature, numerous routing protocols have been proposed for finding QoS paths. In the following sections some of these QoS routing protocols are described.

6.1. CEDAR - The Core Extraction Distributed Ad Hoc Routing (CEDAR) algorithm is proposed as a QoS routing scheme for small to medium-sized ad hoc networks consisting of tens to hundreds of nodes. It dynamically establishes the core of the network, and then incrementally propagates the link states of stable high-bandwidth links to the core nodes. Route computation is on demand, and is performed by the core nodes using only local state. CEDAR has three key components:

Core extraction: A set of nodes is elected to form the core that maintains the local topology of the nodes in its domain, and also to perform route computations. The core nodes are elected by approximating a minimum dominating set 1 of the ad hoc network.

Link state propagation: QoS routing in CEDAR is achieved by propagating the bandwidth availability information of stable links to all core nodes. The basic idea is that the information about stable high bandwidth links can be made known to nodes far away in the network, while information about the dynamic or low bandwidth links remains within the local area.

Route computation: Route computation first establishes a core path from the domain of the source to the domain of the destination. Using the directional information provided by the core path, CEDAR iteratively tries to find a partial route from the source to the domain of the furthest possible node in the core path satisfying the requested bandwidth. This node then becomes the source of the next iteration. In the CEDAR approach, the core provides an efficient low-overhead infrastructure to perform routing, while the state propagation mechanism ensures availability of link state information at the core nodes without incurring high overheads.

\subsection{Multipath Routing Protocol (MRP)}

MRP is a reactive on-demand routing Protocol which extends DSR protocol to find multipath routing coupled with bandwidth and reliability constraint. It consists of three phases: routing discovery, routing maintenance and traffic allocation. In routing discovery phase, the protocol selects several multiple alternate paths which meet the QoS requirements and the ideal number of multipath routing is achieved to compromise between load balancing and network overhead. In routing maintenance phase, it can effectively deal with route failures similar to DSR. Furthermore, the per-packet granularity is adopted in traffic allocation phase.

\subsection{Genetic Algorithm-Based QoS Routing Protocol for MANETS (GAMAN)}

A Genetic Algorithm-based source-routing Protocol for MANETs (GAMAN) is proposed, which uses end-to-end delay and transmission success rate for QoS metrics. Genetic Algorithms 
(GAs) may be employed for heuristically approximating an optimal solution to a problem, in this case finding the optimal route based on the two QoS constraints mentioned.

The first stage of the process involves encoding routes so that a GA can be applied; this is termed gene coding. For this purpose, paths are discovered on-demand and then a network topology view is constructed in a logical tree-like structure. Each node stores a tree routed at itself with its neighbor nodes as child nodes and in turn their neighbor nodes as their children.

The route discovery algorithm is assumed to collect locally computed metrics such as average delay over a link and the link reliability for the links on each path. After the gene encoding stage, the fitness $T$ of each path is calculated as follows:

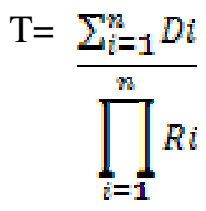

where $D i$ and $R i$ are the delay and reliability of link $i$, respectably. The fitness values are used to select paths for cross-over breeding and mutation operations. The fittest path (with the smallest $T$ ) and the offspring from the genetic operations are carried forward into the next generation. While this method is a useful heuristic for approximating the optimal value over the delay and link reliability metrics at the same time, it requires many paths to be searched in order to collect enough "genetic information" for the GA operations to be meaningful. This means that the method is not suited to large networks.

\subsection{Predictive Location-Based QoS Routing in Mobile Ad Hoc Networks (PLBQR)}

It is a location aware QoS routing protocol in which a location-delay prediction scheme, based on a location-resource update protocol has been performed. The location updates contain resource information pertaining to the node sending the update. This resource information for all nodes in the network and the location prediction mechanism are together used in the QoS routing decisions. There are dynamic changes in topology and resource availability due to the high degree of mobility of nodes in the ad hoc network. Due to these changes, the topological and routing information used by current network protocols is rendered obsolete very quickly.

The advantage of this systems is the prediction of new location based on previous location is made when there is variation in the geographical location. QoS routing based on the resource availability at the intermediate nodes in the source to destination route is performed which is rare in other location based routing scheme. But, accurate prediction on velocity and direction is not made when there are dynamic changes in the direction. The transmission is made only in linear pattern (i.e., angular velocity is kept as zero)

\subsection{QoS Multicast Routing Protocol with Dynamic group topology (QMRPD)}

The QMRPD is a hybrid protocol which attempts to significantly reduce the overhead of constructing a multicast tree with multiple QoS constraints. In QMRPD, a multicast group member can join or leave a multicast session dynamically, which should not disrupt the multicast tree. It satisfies the multiple QoS constraints and least cost's (or lower cost) requirements. Its main objective is to construct a multicast tree that optimizes a certain objective function (e.g., making 
effective use of network resources) with respect to performance-related constraints (e.g., end-toend delay bound, inter-receiver delay-jitter bound, minimum bandwidth available, and maximum packet-loss probability) and design a multicast routing protocol with dynamic group topology. It attempts to minimize the overall cost of the tree. The dynamic group membership has been handled by this protocol with less message processing overhead.

\subsection{QoS Optimized Link State Routing (QOLSR)}

QOLSR protocol which is an enhancement of the OLSR routing protocol to support multiple-metric routing criteria. OLSR is a proactive routing protocol, which inherits the stability of a link state algorithm. The basic QoS metrics considered here are throughput and delay. The routes are immediately available when needed. The OLSR protocol uses a kind of Dijkstra's shortest path algorithm to provide optimal routes in terms of number of hops. It minimizes the control overhead involved in flooding routing information. In which MAC protocol is required to notify the routing protocol when it transmits a packet. QOLSR does not rely on the MAC protocol to provide residual channel capacity or delay information. These values are estimated statistically, using the periodic HELLO messages.

\subsection{Ad hoc QoS on-demand routing (AQOR)}

This protocol uses limited flooding to discover the best route available in terms of smallest end-to-end delay with bandwidth guarantee. A route request packet includes both bandwidth and end-to-end delay constraints. Let $\mathrm{T}_{\max }$ denote the delay constraint. If a node can satisfy both constraints, it will rebroadcast the request to the next hop and switch to explore status for a short period of $2 \mathrm{~T}_{\max }$.

If multiple request packets arrive at the destination, it will send back a reply packet along each of these routes. Intermediate nodes will only forward the reply, if they are still in explored state. However, the bandwidth reservation for each flow is only activated by the arrival of the first data packet from the source node. Delay is measured during route discovery. The route with the least delay is chosen by the source. No mechanism for connection tear-down is needed or integrated, since all reservations are only temporary. Timers are reset every time a route is used. So there is an upper time bound after which broken routes are detected.

To further reduce communication overhead during route discovery, AQOR can work with some location aided routing protocols. For delay violation detection, the estimated time offset between the systems clocks of source and destination node has to be known.

\section{Summary OF QoS routing Protocols}

To facilitate a comparison among the different QoS-aware routing protocols, the salient features of the QoS routing protocols is described in a table. The table lists the design constraints listed earlier such as Route discovery, Resource reservation, Route maintenance, QoS metrics constrained, Network architecture and routing overhead and discussing how each protocol addresses. 


\begin{tabular}{|l|l|l|l|l|l|l|}
\hline $\begin{array}{l}\text { Routing } \\
\text { protocol }\end{array}$ & $\begin{array}{l}\text { Network } \\
\text { architecture }\end{array}$ & $\begin{array}{l}\text { Route } \\
\text { discovery }\end{array}$ & $\begin{array}{l}\text { Type of } \\
\text { QoS } \\
\text { guarantee }\end{array}$ & $\begin{array}{l}\text { Resource } \\
\text { reservation }\end{array}$ & $\begin{array}{l}\text { QoS } \\
\text { metrics }\end{array}$ & $\begin{array}{l}\text { Routing } \\
\text { overhead }\end{array}$ \\
\hline CEDAR & Hierarchical & $\begin{array}{l}\text { Proactive/ } \\
\text { Reactive }\end{array}$ & Soft & Yes & Bandwidth & core setup \\
\hline MRP & Hierarchical & Reactive & Soft & Yes & Bandwidth & $\begin{array}{l}\text { Full flooding } \\
\text { of RREQ }\end{array}$ \\
\hline GAMAN & Hierarchical & Reactive & Soft & Yes & $\begin{array}{l}\text { Bounded } \\
\text { delay, loss } \\
\text { packet loss } \\
\text { rate }\end{array}$ & $\begin{array}{l}\text { Node } \\
\text { traversal } \\
\text { delay and }\end{array}$ \\
\hline PLBQR & $\begin{array}{l}\text { Location } \\
\text { prediction }\end{array}$ & $\begin{array}{l}\text { Proactive/ } \\
\text { Reactive }\end{array}$ & Soft & No & $\begin{array}{l}\text { Delay, and } \\
\text { Bandwidth }\end{array}$ & $\begin{array}{l}\text { Route } \\
\text { recomputatio } \\
\text { nanticipation } \\
\text { of } \\
\text { breakage }\end{array}$ \\
\hline QMRPD & Hierarchical & Reactive & $\begin{array}{l}\text { Pseudo- } \\
\text { hard }\end{array}$ & Yes & $\begin{array}{l}\text { Bandwidth, } \\
\text { Delay, } \\
\text { Delay-jitter } \\
\text { and cost }\end{array}$ & $\begin{array}{l}\text { Less message } \\
\text { processing } \\
\text { overhead }\end{array}$ \\
\hline QOLSR & Hierarchical & Proactive & Soft & Yes & $\begin{array}{l}\text { Throughput } \\
\text { and Delay }\end{array}$ & $\begin{array}{l}\text { Minimum } \\
\text { flooding of } \\
\text { RREQ }\end{array}$ \\
\hline AQOR & Flat & Reactive & Soft & Yes & $\begin{array}{l}\text { Bandwidth, } \\
\text { Delay }\end{array}$ & $\begin{array}{l}\text { Full flooding } \\
\text { of RREQ }\end{array}$ \\
\hline FAODV & Flat & Reactive & Soft & Yes & $\begin{array}{l}\text { Bandwidth, } \\
\text { Delay }\end{array}$ & $\begin{array}{l}\text { Minimum } \\
\text { flooding of } \\
\text { RREQ }\end{array}$ \\
\hline
\end{tabular}

Table 1. Comparison of QoS aware routing protocols

\section{Future Challenges}

MANETs are likely to expand their applications in the future communication environments. The support for QoS will thus be an important and desirable component of MANETs. Several important research issues and open questions need to be addressed to facilitate QoS support in MANETs. It includes admission control policies and protocols, QoS preservation under failure conditions, QoS support for multicast operations and security against denial-of service attack etc. Power control and accommodating multiple classes of traffic requires further research attention.

\section{SUMMARY}

In this paper, several issues and challenges involved in providing QoS were discussed. A thorough overview of QoS metrics and design considerations is also provided. Then the existing QoS approaches were classified according to several criteria such as interaction between network and MAC layer, type of QoS guarantee assured, QoS metrics focused and route updating 
mechanism etc. We have summarized the operation, strengths and drawbacks of existing QoS routing approaches in order to expose the current trends of progress in the field and to identify the topics for further research.

\section{References}

[1] C.R.Lin and J.S. Liu., "QoS routing in ad hoc wireless networks", IEEE J.Select.Areas Commun., vol.17, pp.1488-1505, 1999.

[2] C.Perkins, "Ad-hoc On-Demand Distance Vector (AODV) routing", RFC3561[S], 2003.

[3] D.B.Johnson, D.A.Maltz, Y.C.Hu, "The Dynamic Source Routing protocol for mobile ad hoc networks", Internet Draft, 2004.

[4] J.Hong, "Efficient on-demand routing for mobile ad hoc wireless access networks", IEEE journal on selected Areas in Communications 22(2004), 11-35.

[5] Luo Junhai, Xue Lie and Ye Danxia "Research on multicast routing protocols for mobile ad hoc networks" Computer Networks52(2008), 988-997.

[6] S. Chen, Routing Support for Providing Guaranteed End-to-End Quality-of-Service, Ph.D Thesis, University of IL at Urbana-Champaign, 1999.

[7] S. Chakrabarti and A. Mishra, "QoS issues in ad-hoc wireless networks" IEEE Commun. Mag., vol.39, pp.142-148, Feb. 2001.

[8] J.N. Al-Karaki and A.E.Kamal, "Quality of Service routing in mobile ad hoc networks: Current and future trends" in Mobile Computing Handbook, CRC Publishers, 2004.

[9] T.B.Reddy I.Karthigeyan, B.Manoj and C.S.R.Murthy, "Quality of service provisioning in ad hoc wireless networks: a survey of issures and solutions." Vol.4, pp.83-124, 2006.

[10] S.Saunders, "Antennas and Propagation for Wireless Communication System Concept and design", New York, USA: John Wiley and Sons, 1999.

[11] Y.Luo, J.Wang and J.Chen et al., "Algorithm based on mobility prediction and probability for energy efficient multicasting in ad-hoc networks" Computer research and development vol.43(2), pp.231-237, 2006.

[12] Y.Yang and R.Kravets "Contention-aware admission control for ad hoc networks", IEEE Trans.Mobile Comput., vol.4, 363-377, 2005.

[13] C.R.Lin and J.S. Liu., "QoS routing in ad hoc wireless networks", IEEE J.Select.Areas Commun., vol.17, pp.1426-11438, 1999.

[14] S.Chen and K.Nahrstedt, "Distributed quality-of-service routin in ad hoc networks" IEEE J.Select.Areas Commun., vol.17, pp.1488-1505, 1999.

[15] A.R.Bashandy, E.K.P.Chong and A.Ghafoor, "Generalized quality-of-service routing with resource allocation" IEEE J.Select.Areas Commun., vol.23, pp.450-463, 2005.

[16] A.Abradou and W.Zhuang, "A position based QoS routing scheme for UWB mobile ad hoc networks" IEEE J.Select.Areas Commun., vol.24, pp.850-856, 2006.

[17] L.Li, C.Li, "A hierarchical QoS multicast routing protocol for mobile ad-hoc networks", Chinese Journal of Electronics 15(4) (2006), 573- 577.

18] M.S.Kumar, C.Venkatesh, A.M.Ntarajan, "Performance comparison of multicast protocol for physically hierarchical ad-hoc networks using neural Concepts", $7^{\text {th }}$ International conference on Signal Processing Proceedings ICSP, 2004, 1581-1584.

[19] R.Sivakumar, P.Sinha and V.Bharghavan, "CEDAR: a core-extraction distributed ad hoc routing algorithm" IEEE J.Select.Areas Commun., vol.17, pp.1454-1465, 1999.

[20] L.A.Latif, A.Alliand, C.C. Ooi, "Location based geocasting and forwarding (LGF) routing protocol in mobile-adhoc network", Proceedings on the Advanced Industrial Conference on Telecommunications/Service assurance with partial Intermittent Resources (2005) 536-541.

[21] K.Chen, K.Nahrstedt, "Effective location guided-tree construction algorithms for small group multicast in MANET", Proceedings of the INFOCOM (2002) 1180-1189.

[22] M.Transier, H.Fubler, J.Widmet et al., "Scalable position based multicst for mobile ad-hoc networks" in:Proceddings of the First International; workshop on Broadband wireless multimedia: Algorithms, Arcgutectures, and applications, San Jose, CA, Oct. 2004. 
[23] H.Badis and K.A.Agha, "QOLSR, QoS routing for ad hoc wireless networks using OLSR", Wiley European Trans. Telecomunications, vol.15(4), pp.427-422, 2005.

[24] S.H.Shah, K. Nahrstedt, "Predictive location-based QoS routing in mobile ad-hoc networks", in: Proceeding of IEEE ICC 2002, vol.2, pp.1022-1027, 2002.

[25] E.M. Royer C.E.Perkins, A $>$ R $>$ Das "Quality of service for ad-hoc on-demand distance vector routing”, IETF Internet draft, July 2000.

[26] B.Kaliaperumal, A.Ebenezer, Jeyakumar, "Adaptive Core-based scaleable multicasting networks", INDICON, Annual IEEE (2005), 198-202.

[27] H.Dhillon, H.Q.Ngo, "CQMP: a mesh-based multicast routing protocol with consolidated query packets", in: IEEE wireless communications and networking conference WCNC 2005, pp.2168-2174.

[28] J.Biswas, M.Barai, S.K.Nandy, "Efficient hybrid multicast routing protocol for ad-hoc wireless networks, local computer networks", in: 29 ${ }^{\text {th }}$ Annual IEEEE International Conference on Nov.2004, pp.180187.

[29] M.Sheng, J.Li and Y.Shi, "Routing protocol with QoS guarantees for ad-hoc network" Electronic Letters, vol.39, pp.143-145, 2003.

[30] R.Gupta, Z.Jia, T.Tung and J.Walrand, "Interference aware QoS routing for ad-hoc networks" in Proc.IEEE Canadian Conf. on Electrical and Computer Engineering, vol.3, pp.1535-1538, 2003.

[31] H.Shen, B.Shi, L.Zou etal., "A distributed entropy based long-life QoS ruting algorithm in ad-hoc network", IEEE J.Select.Areas Commun., vol.17, pp.1454-1465, 1999.

[32] J.Stine and G.de Veciana, "A paradigm for QoS in wireless ad hoc networks using synchronous signaling and node state”, IEEE J.Select.Areas Commun., vol.17, pp.1454-1465, 1999.

[33] Li Layuan and Li Chunlin, "A QoS Multicast Routing Protocol for Dynamic group topology", Information Sciences 169 (2005) 113-130.

[34] L.Barolli, A.Koyama et al., "A QoS routing method for ad-hoc networks based on genetic algorithm", in Proc. $14^{\text {th }}$ Int.Wksp. Database and Expert Systems Applications, pp.175-179, 2003.

[35] D.Kim, C.H.Min et al., "On-demand SIR and bandwidth guaranteed routing with transmit power assignment in ad hoc mobile networks" IEEE Trans. Veh. Technol., vol.53, pp.1215-1223, 2004. 